\title{
Phase separation in the Edwards model
}

\author{
S. Ejima, ${ }^{1}$ S. Sykora ${ }^{2}$ K. W. Becker ${ }^{3}$ and H. Fehske ${ }^{1}$ \\ ${ }^{1}$ Institut für Physik, Ernst-Moritz-Arndt-Universität Greifswald, 17487 Greifswald, Germany \\ ${ }^{2}$ Institute for Theoretical Solid State Physics, IFW Dresden, 01069 Dresden, Germany \\ ${ }^{3}$ Institut für Theoretische Physik, Technische Universität Dresden, 01062 Dresden, Germany
}

(Dated: November 21, 2018)

\begin{abstract}
The nature of charge transport within a correlated background medium can be described by spinless fermions coupled to bosons in the model introduced by Edwards. Combining numerical density matrix renormalization group and analytical projector-based renormalization methods we explore the ground-state phase diagram of the Edwards model in one dimension. Below a critical boson frequency any long-range order disappears and the system becomes metallic. If the charge carriers are coupled to slow quantum bosons the Tomonaga-Luttinger liquid is attractive and finally makes room for a phase separated state, just as in the $t$ - $J$ model. The phase boundary separating the repulsive from the attractive Tomonaga-Luttinger liquid is determined from long-wavelength charge correlations, whereas fermion segregation is indicated by a vanishing inverse compressibility. On approaching phase separation the photoemission spectra develop strong anomalies.
\end{abstract}

\section{PROBLEM}

The Edwards fermion-boson mode ${ }^{1,2}$ constitutes a paradigmatic model for the theoretical description of quantum transport in solids. Charge transport normally takes place in the presence of some background medium $\underline{3}^{3}$ Examples for such a "background" could be a spin-,

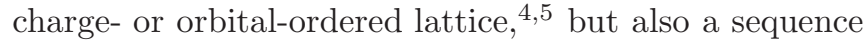
of chemical side groups along the transport path, a deformable medium, or even a heat bath might be possible. In all these cases, the transfer of the charge carriers will be strongly influenced by fluctuations, which may exist in the background medium. The other way around, the properties of the background are quite often determined by the motion of the particle itself.

Correlations inherent in such a complex interactive system are mimicked by a boson-affected hopping of spinless fermionic particles in the Edwards model. It reads:

$$
H=-t_{b} \sum_{\langle i, j\rangle} f_{j}^{\dagger} f_{i}\left(b_{i}^{\dagger}+b_{j}\right)-\lambda \sum_{i}\left(b_{i}^{\dagger}+b_{i}\right)+\omega_{0} \sum_{i} b_{i}^{\dagger} b_{i} .
$$

Every time a fermion $f_{i}^{(\dagger)}$ hops, it creates or absorbs a boson $b_{i}^{(\dagger)}$ of energy $\omega_{0}$ at the sites it leaves or enters. Such an excitation or de-excitation corresponds to a local "distortion" of the background. Because of quantum fluctuations the distortions are able to relax $(\propto \lambda)$. The physically most interesting regimes in this setting are those of vanishing fermion density and of a half-filled band, representing doped Mott insulators, polaronic organics and charge-density-wave (CDW) systems ${ }^{6}-\frac{9}{2}$ with possible relevance to high- $T_{c}$ superconductors, 10,11 colossal magnetoresistive manganites,, $12-14$ carbon nanotubes, $, 15,16$ graphene $\frac{17,18}{}$ and CDW transition metal complexes, respectively. However, the Edwards model also reveals fascinating properties over the whole density range.

On these grounds, the main goal of the present work is to pinpoint the ground-state phase diagram of the onedimensional (1D) Edwards model as a function of the band filling $n$. Thereby we demonstrate that this model indeed captures a number of very interesting phenomena, including e.g. electronic phase separation (PS). To obtain reliable information about the ground-state and spectral properties of the model in the thermodynamic limit, we employ numerical pseudosite density-matrix renormalization group (DMRG) and dynamical DMRG (DDMRG) techniques (supplemented by a careful finite-size scaling procedure, for details see Refs. 9, 21 24), in combination with the analytical projective renormalization method (PRM) 25,26

So far the 1D Edwards model has been solved exactly by numerical approaches for two cases. $22,24,27$ The first, in no way trivial case concerns just a single particle on an infinite lattice, where - depending on the model parameters - transport appears to be quasi-free, diffusive, or boson-assisted $\stackrel{6.28}{ }$ When strong correlations develop at small $\lambda$ and large $\omega_{0}$, the background becomes "stiff" and the spinless particle's motion resembles those of a hole in an antiferromagnetic spin background, $, 29,30$ as known from the $t-J$ model. Interestingly, the Edwards model allows for so-called Trugman paths ${ }^{31}$ in an 1D setting (Trugman paths usually unwind the string of misaligned spins a mobile hole leaves behind in two dimensions). The second case is half-filling $n=1 / 2$. Here a metal-insulator transition has been proven to exist: For small $\lambda$ and large $\omega_{0}$ the repulsive Tomonaga-Luttinger liquid (TLL) gives way to a CDW $\stackrel{7,8}{?}$ The CDW constitutes a few-boson state that typifies a Mott-Hubbard insulator rather than a Peierls state (normally established by the softening of a boson mode).

\section{THEORETICAL RESULTS}

\section{A. Ground-state properties}

The situation at finite density $n(n \neq 1 / 2)$ is much less understood. By analogy with the $t-J$ model one might expect that the system is metallic for $0<n<1 / 2$, 
at least if the background is not too stiff. If so, the next question will be, whether the Edwards model might support the pairing of electrons in a certain parameter regime $\left(\lambda, \omega_{0}\right)$. Apparently a second electron, following the path of a first one, can take advantage of the background excitations (bosons) left behind by the first electron. This acts like an effective attractive interaction. If this attraction completely dominates the kinetic energy the system might even phase-separate into particleenriched and particle-depleted regions $\stackrel{32}{ }$ Since the Edwards model is not particle-hole symmetric, it is a moot point whether a corresponding hole pairing mechanism is at play also for $1 / 2<n<1$.

To address these problems, we analyze the charge correlations existing in the ground-state of the 1D Edwards model. Firstly, we calculate the charge structure factor

$$
S_{c}(q)=\frac{1}{L} \sum_{j, l} e^{i q(j-l)}\left\langle\left(f_{j}^{\dagger} f_{j}-n\right)\left(f_{l}^{\dagger} f_{l}-n\right)\right\rangle,
$$

for a system with $N$ fermions, $L$ sites, and open boundary conditions (OBC). So the particle density is $n=N / L$ and the momenta $q=2 \pi s / L$ with integers $0<s<L$. The TLL charge exponent is proportional to the slope of $S_{c}(q)$ in the long-wavelength limit $q \rightarrow 0^{+}$(cf. Refs. $\underline{33}$ and 34$)$ :

$$
K_{\rho}=\pi \lim _{q \rightarrow 0} \frac{S_{c}(q)}{q} .
$$

Then, $K_{\rho}>1\left(K_{\rho}<1\right)$ characterizes an attractive (repulsive) TLL for our spinless fermion transport model (11), and $K_{\rho}=1 / 2$ will define a metal-insulator transition point at $n=1 / 2, \frac{35}{2}$ That the finite-size scaling of $S_{c}(q)$ and $K_{\rho}$ works well has been demonstrated for the half-filled band case $\stackrel{\underline{\underline{8}}}{\mathrm{~S}}$ Secondly, in order to comprise potential PS, we determine the finite-size equivalent of the charge compressibility $\underline{\underline{36}}$

$$
\kappa=\frac{L}{N^{2}}\left[\frac{E_{0}(N+2)+E_{0}(N-2)-2 E_{0}(N)}{4}\right]^{-1},
$$

with $E_{0}(N)$ being the ground-state energy for $N$ electrons on $L$ sites. An infinite compressibility signals the formation of a PS state.

Fig. 1 demonstrates that all this works in practice. Exemplarily choosing $n=1 / 8$ and $\lambda=0.2$, we show how the TLL exponent $K_{\rho}$ and the inverse compressibility $\kappa^{-1}$ scale with increasing system size at various $\omega_{0}$. The transition point between a repulsive TLL - obviously realized at $\omega_{0}>\omega_{0, c 1}$ when excitations of the background are energetically rather costly - and an attractive TLL at smaller $\omega_{0}$, can be read off from the inset [depicting the extrapolations $K_{\rho}(L \rightarrow \infty)$ ] to be $\omega_{0, c 1}(\lambda=0.2) \simeq 1.118$. Reducing $\omega_{0}$ further, in the attractive TLL phase, a dramatic increase in $K_{\rho}$ is observed at $\omega_{0, c 2} \simeq 0.6$. Our inverse compressibility data indicate that at this point the attraction among the particles becomes so strong that the system shows PS, i.e., $\kappa^{-1}=0$ for $\omega_{0}<\omega_{0, c 2}$.
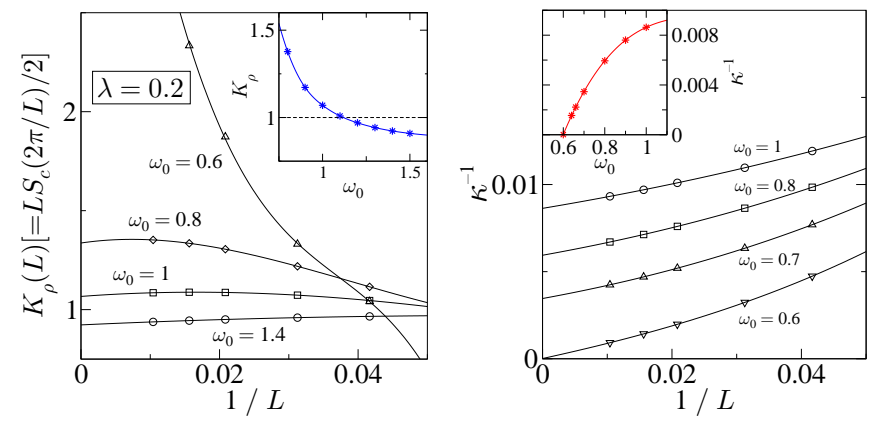

Figure 1. Finite-size scaling of the TLL parameter $K_{\rho}$ (left panel) and inverse compressibility $\kappa^{-1}$ (right panel) for the $1 \mathrm{D}$ Edwards model with $\lambda=0.2$ at $n=1 / 8$. Insets show the variation with $\omega_{0}$ of the $L \rightarrow \infty$ extrapolated values. Results are obtained by DMRG for a lattice with $L$ sites and OBC. In all DMRG runs we take into account up to $n_{b}=$ 4 bosonic pseudosites and determine $n_{b}$ by the requirement that the local boson density of the last pseudosite is less than $10^{-6}$ for all $j$. Using selectively $n_{b}=5$, we controlled that convergence is truly achieved. Furthermore we keep up to $m=1200$ density-matrix eigenstates in the renormalization steps to ensure that the discarded weight is smaller than $10^{-8}$.

Proceeding in the same manner for different values of $n$ and $\lambda$ respectively $\omega_{0}$, we can map out the phase diagram of the 1D Edwards model. The outcome is displayed in Fig. 22 Let us first consider the case of a not too small boson relaxation $(\propto \lambda)$, which ensures that the system is metallic for large $\omega_{0}$ in the whole density regime. Then, as the upper panel of Fig. 2 shows, depending on $n$ we find up to three different regimes: For small and large particle densities a repulsive TLL, an attractive TLL and a PS state appear in sequence as the energy of the bosons is lowered. In contrast, around half-filling only the repulsive TLL exists. At this point we would like to remind the readers that at half-filling, for smaller values of $\lambda$, $\left(\lambda<\lambda_{c}\right)$, a CDW is formed for $\omega_{0}>\omega_{0, c} \stackrel{\underline{8}}{\text {. }}$ Our unbiased DMRG calculations give no evidence for any other phases. Note that the behavior in the low-density regime is consistent with what is found for the $1 \mathrm{D} t-J$ model ${ }^{36.37}$, where the holes correspond to the spinless fermions in the Edwards model. As mentioned before, the phase diagram is not symmetric with respect to $n=1 / 2$. This is because the hopping of a hole (missing electron) to a neighboring site creates in the Edwards model a boson at the arrival site and not on the departure site as in the motion of a single particle. Since this boson can be destroyed immediately when the hole makes a further hop, there is no string effect and a few holes propagate more easily than a few electrons. Hence the background at fixed $\omega_{0}$ and $\lambda$ appears to be less stiff for $n \lesssim 1$ than for $n \gtrsim 0$ and, as a result, the boundary between the repulsive and attractive TLL is shifted to larger values of $\omega_{0, c 1}$. Contrariwise, since carriers will be less mobile in a state with charge separation, the boundary between the attractive TLL and the PS state $\omega_{0, c 2}$ is shifted to smaller values if one compares the corresponding results at high and low 
carrier density.

We next consider a fixed particle density $n=1 / 8$ and track the phase boundaries in the $\lambda-\omega_{0}$ plane; see the lower panel of Fig. 2. The repulsive TLL established for large $\omega_{0}$ is strongly renormalized if the ability of the background to relax is low. For example, we find $K_{\rho}=0.527$ for $\omega_{0}=2$ at $\lambda=0.01$. Lowering $\omega_{0}$ for such a stiff background, the transition to the PS state happens almost instantaneously with a narrow intervening attractive TLL state; at $\lambda=0.01$ we have $\omega_{0, c 1} \simeq 0.747$ and $\omega_{0, c 2} \simeq 0.672$. If we fix, on the other hand, $\omega_{0}=2$ and increase $\lambda$, we observe a strong enhancement of $K_{\rho}$ in the interval $0<\lambda<0.3$ ( $K_{\rho}=0.767,0.879,0.932$ for $\lambda=0.1,0.2,0.3$, respectively), followed by a very gradual increase until the transition to the attractive TLL takes place at about $\lambda_{c 1} \simeq 1$. Obviously, the region where the attractive TLL (PS state) constitutes the ground state expands (shrinks) as $\lambda$ gets larger, which can be traced back to the subtle competition between kinetic energy and charge segregation effects.

To gain deeper insight into the different mechanisms at play, we analyze the variation of the local fermion $\left[n_{j}^{f}=\left\langle f_{j}^{\dagger} f_{j}\right\rangle\right]$ and boson $\left[n_{j}^{b}=\left\langle b_{j}^{\dagger} b_{j}\right\rangle\right]$ densities in real space. In Fig. 3 we show both density profiles for $n=1 / 8$ and characteristic values of $\lambda$ and $\omega_{0}$, implementing repulsive TLL, attractive TLL, and PS states. In the metallic regime, the OBC lead to Friedel oscillations in the particle density. These oscillations are especially pronounced for the repulsive TLL [panel (a)], and they will be even stronger for $\omega_{0}=2$ (not shown), where the number and fluctuations of the bosons is reduced. Note that the Friedel oscillations caused by the OBC will be algebraically reduced, if we move for very large systems towards the central part of the chain. If we enter the attractive TLL regime by increasing $\lambda$ at fixed $\omega_{0}$ the Friedel oscillation will be smeared out [panel (b)]. Thereby, the number of oscillations stays constant, which means that a pairing of electrons does not occur. This is an important difference from the spinful 1D $t-J$ model, where a recent DMRG study reveals that the number of Friedel oscillations is halved (by increasing $J$ ), corresponding to half the number of particles, which indicates pairing: $\stackrel{37}{\text { Next }}$ lowering $\omega_{0}$ to a point where PS sets in, the particle density oscillations vanish, see panel (c). There, just above the PS boundary, no evidence is found for the clustering of multiple particles in several groups. This agrees with

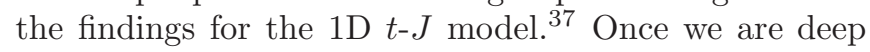
inside the PS region, a density distribution results, where a single island of particles at the central part of the system appears, leaving a sizable number of almost empty sites at both ends of the chain [see panel (d)]. Looking at the bosonic degrees of freedom, we see that the strong attraction among these particles is mediated by a boson cloud covering the electron-rich region. As a result the kinetic fluctuations will be strongly quenched.

It is all but impossible to comprise by DMRG the large number of bosons and the strong bosonic fluctuations, which appear at still smaller $\omega_{0}$ in the Edwards model,
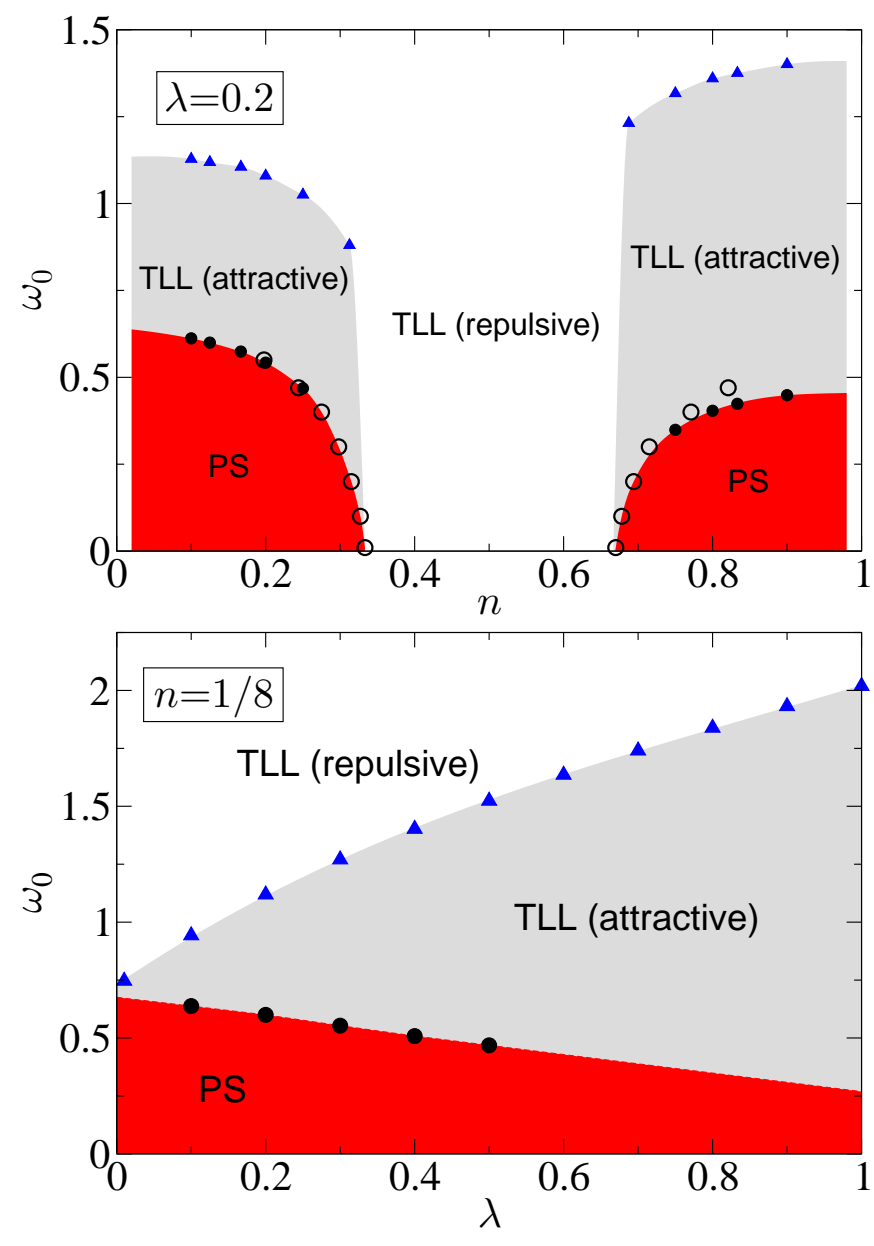

Figure 2. Ground-state phase diagram of the 1D Edwards model, showing repulsive TLL $\left(K_{\rho}<1\right)$, attractive TLL $\left(K_{\rho}>1\right)$, and PS $\left(\kappa^{-1}=0\right)$ regions. The phase boundaries were obtained by DMRG (filled symbols) in the course of a careful finite-size scaling analysis and for the infinite system directly by PRM (open symbols). The upper panel displays the phase diagram as a function of $n$, varying $\omega_{0}$ at fixed $\lambda=0.2$; the lower panel gives the phase diagram in the $\lambda-\omega_{0}$ plane for fixed density $n=1 / 8$.

simply because the dimension of the Hilbert space increases dramatically. To access the low- $\omega_{0}$ regime and reconfirm the DMRG phase boundaries, obtained for the 1D Edwards model at larger values of $\omega_{0}$, we employ the analytical PRM approach $\underline{25}$ The basic idea of the PRM is to construct-performing a sequence of discrete unitary transformations and eliminating all transitions with energy larger than a given cutoff energy - an effective non-interacting Hamiltonian $\tilde{H}$ with renormalized parameters (in the limit of vanishing cutoff energy). For the metallic state of the Edwards model, in momentum space, it takes the form

$$
\tilde{H}=\sum_{k} \tilde{\varepsilon}_{k} f_{k}^{\dagger} f_{k}+\sum_{q} \tilde{\omega}_{q} b_{q}^{\dagger} b_{q}+\tilde{E},
$$

where the renormalization equations for $\tilde{\varepsilon}_{k}$ and $\tilde{\omega}_{q}$ have 

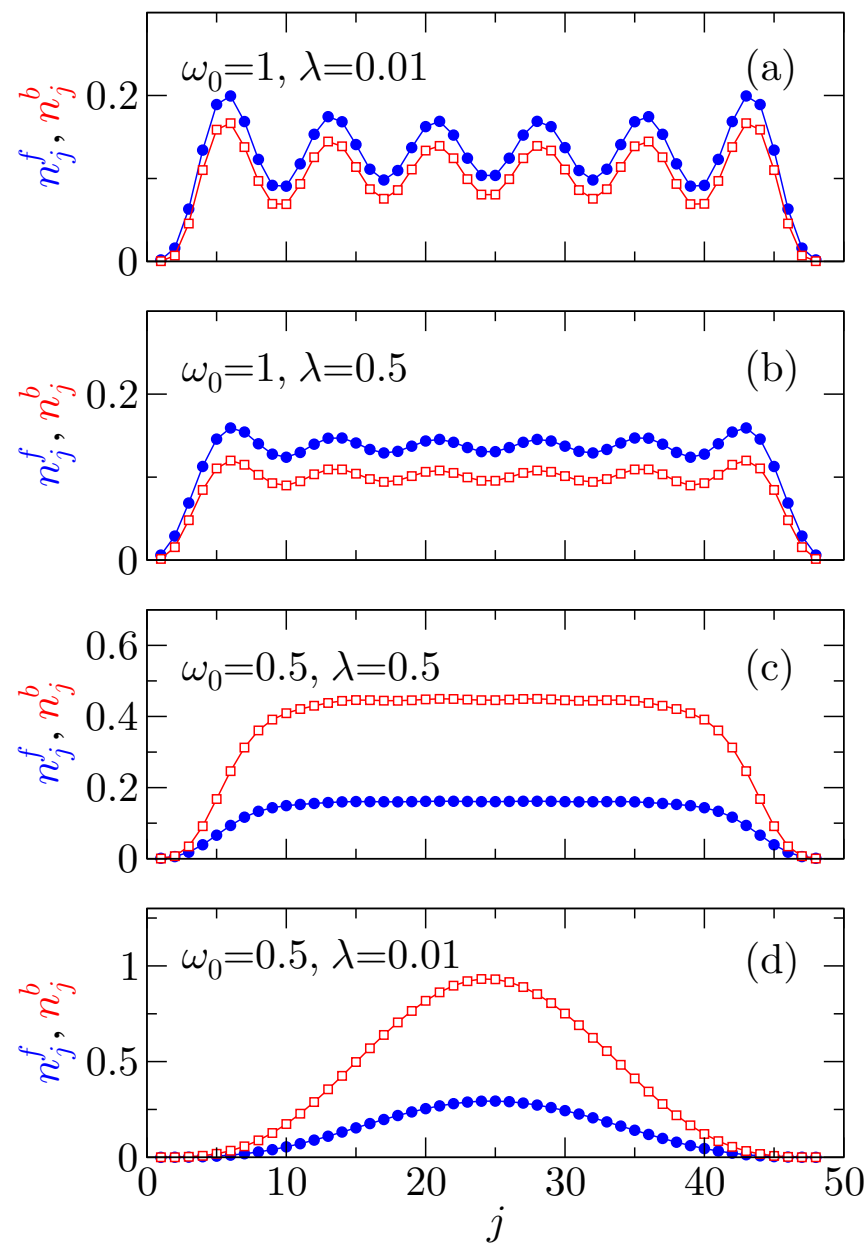

Figure 3. Local particle (filled circles) and boson (open squares) densities for the $1 \mathrm{D}$ Edwards model with $n=1 / 8$, calculated for a 48 -site system with OBC at various $\lambda$ and $\omega_{0}$.

been derived in Ref. 26 (for the half-filled band case). In order to fix the boundary between metallic and PS states, we (i) solve the renormalization equations at a given $E_{F}$ in the TLL phase, (ii) determine the corresponding fermion density $n$, and (iii) slightly vary $E_{F}$ (to get closer to the PS instability), determine $n$, and repeat the whole procedure self-consistently until the functional dependence of $n$ on $E_{F}$ is established. A vanishing inverse compressibility can then be read off from a sudden jump of $n$ under a tiny variation of $E_{F}$ or, the other way around, if a plateau in the $E_{F}$ versus $n$ plot appears. The transition points, determined in the manner described, were inserted in Fig. 2 to complete the ground-state phase diagram in the $n-\omega_{0}$ plane. Whenever points can be compared we find a remarkable agreement between our DMRG and PRM data. The deviation of the PRM data at larger particle densities $n \gtrsim 0.75$ results from uncertainties in fixing the jump of $n$ under $E_{F}$ variation. Here we should trust in the DMRG phase boundary.

\section{B. Spectral properties}

Let us finally discuss the single-particle spectrum of the 1D Edwards model. The single particle excitations associated with the injection $(+)$ or emission $(-)$ of an electron with wave vector $k$,

$$
A^{ \pm}(k, \omega)=\sum_{m}\left|\left\langle\psi_{m}^{ \pm}\left|f_{k}^{ \pm}\right| \psi_{0}\right\rangle\right|^{2} \delta\left(\omega \mp \omega^{ \pm}\right),
$$

can be computed by DDMRG,$\stackrel{23,24}{\underline{2}}$ where $f_{k}^{+}=f_{k}^{\dagger}, f_{k}^{-}=$ $f_{k},\left|\psi_{0}\right\rangle$ is the ground state of a $L$-site system in the $N$ particle sector, and $\left|\psi_{m}^{ \pm}\right\rangle$denote the $m$-th excited states in the $(N \pm 1)$-particle sectors with excitation energies $\omega_{m}^{ \pm}=E_{m}^{ \pm}-E_{0}$.

Within PRM we find for the photoemission part

$$
\begin{aligned}
A^{-}(k, \omega)= & \tilde{\alpha}_{k}^{2} \tilde{n}_{k}^{f} \delta\left(\omega-\tilde{\varepsilon}_{k}\right) \\
& +\sum_{q} \tilde{\beta}_{k, q}^{2}\left(1+\tilde{n}_{q}^{b}\right) \tilde{n}_{k+q}^{f} \delta\left(\omega+\tilde{\omega}_{q}-\tilde{\varepsilon}_{k+q}\right) \\
& +\sum_{q} \tilde{\gamma}_{k, q}^{2} \tilde{n}_{q}^{b} \tilde{n}_{k-q}^{f} \delta\left(\omega-\tilde{\omega}_{q}-\tilde{\varepsilon}_{k-q}\right)
\end{aligned}
$$

where $\tilde{n}_{q}^{f}\left(\tilde{n}_{q}^{b}\right)$ are the fermion (boson) occupation numbers in momentum space calculated with the renormalized Hamiltonian $\tilde{H}$. The coefficients $\tilde{\alpha}_{k}^{2}, \tilde{\beta}_{k, q}^{2}$, and $\tilde{\gamma}_{k, q}^{2}$ follow from renormalization equations $\underline{\underline{26}}$ Taking the corresponding expression for $A^{+}(k, \omega)$, the sum rule $\int_{\infty}^{\infty} d \omega\left[A^{+}(k, \omega)+A^{-}(k, \omega)\right]=1$ is fulfilled.

Figure 4 gives the combined photoemission spectrum, $A(k, \omega)=A^{+}(k, \omega)+A^{-}(k, \omega)$, as obtained by DDMRG for quasimomenta $k$. In the repulsive TLL regime (left panel, $\omega_{0}=2$ ) we find a rather coherent signal with comparable spectral weight for all $k$ values $k \lesssim \pi / 2$. For larger $k$, excitations with at least one additional $\omega_{0}$-boson involved become important (recall that both initial $N$ particle- and target $(N \pm 1)$-particle states are multiboson states with a momentum being the total momentum of electrons and bosons). The spectrum in the attractive
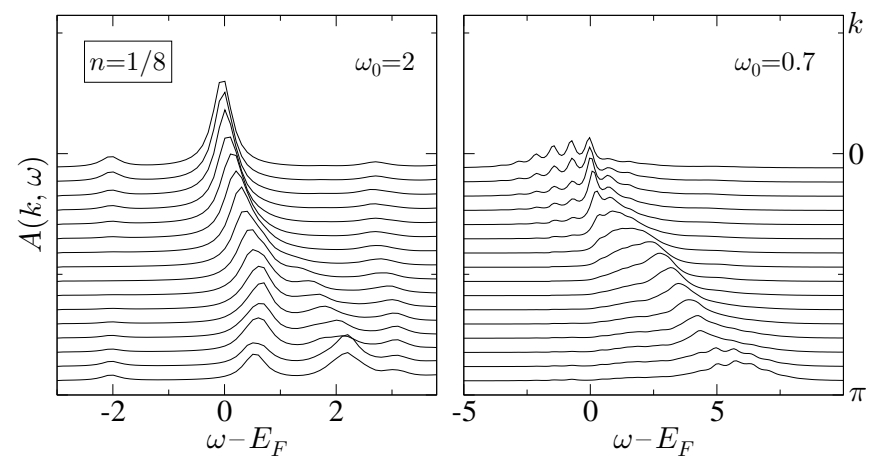

Figure 4. Line-shape of the single-particle spectral function $A(k, \omega)$ for the 1D Edwards model with $\lambda=0.2$ at $n=1 / 8$. Results are obtained by DDMRG for an 16-site chain with quasimomenta $k=\pi s /(L+1)$, using a broadening $\eta=0.2$. 


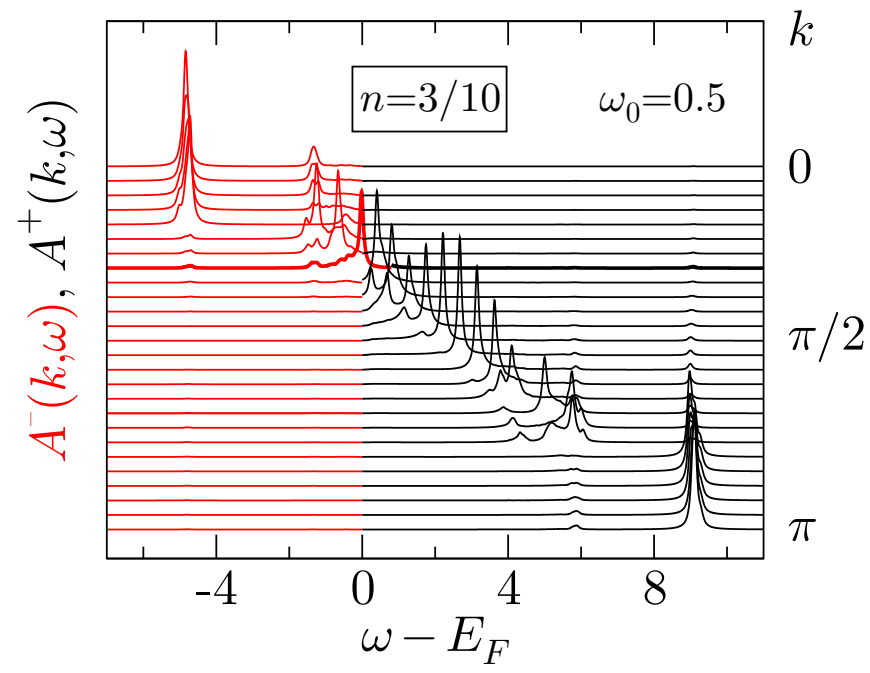

Figure 5. PRM [inverse] photoemisson spectrum $\left[A^{+}(k, \omega)\right]$ $A^{-}(k, \omega)$ for the Edwards model with $\lambda=0.2, n=3 / 10$. The bold line marks the signal at the Fermi momentum $k_{F}=0.3 \pi$.

TLL phase (right panel, $\omega_{0}=0.7$ ) shows a sharp absorption signal in the vicinity of $k_{F}$ only. Here the (inverse) photoemission spectrum for $k<k_{F}\left(k>k_{F}\right)$ exhibits a few absorption maxima at multiples of the boson energy. Obviously, due to the smaller $\omega_{0}$, here the dynamics of the system becomes dominated by bosonic fluctuations.

The (inverse) photoemission close to the transition to the PS state is depicted Fig. 5, as calculated by PRM for $\omega_{0}=0.5$. Here the almost dispersionless signal for $k \leq 0.212 \pi(k \geq 0.788 \pi)$ is a precursor of the PS instability. As we noted above, $E_{F}$ does not show any variation with $n$ if $n$ is smaller (larger) than the lower (upper) crit- ical density $n_{c 2}$ for PS. Since in the PRM derivation of $A^{ \pm}(k, \omega)$ terms with two (and more) bosonic creation or annihilation operators were neglected, $\stackrel{26}{r}$ the photoemission spectrum of Fig. 5 does not feature the multiboson related signatures found within a DDMRG treatment.

\section{CONCLUSIONS}

To summarize, the combination of analytical (PRM) and numerical (DMRG) approaches permits the precise determination of the ground-state phase diagram of the 1D Edwards model in the whole parameter regime. In the low- and high-density regions, the attraction between the particles mediated by the bosonic degrees of freedom representing the background medium might become so strong that electronic PS sets in. In the remaining region the system realizes a TLL or, at $n=1 / 2$, possibly even a truly long-range ordered CDW state. Depending on the properties of the background medium, the TLL might be attractive or repulsive. The richness of the phase diagram is remarkable. The model captures important features of Holstein, $t-J$, Hubbard, and FalicovKimball type models $\underline{\underline{6}-\underline{3}, 38}$ Since the Edwards model is one of the simplest models for studying transport in lowdimensional systems, an inspection of its predictions with ultracold fermion/boson quantum gases,$\frac{39}{3}$ as, e.g., carried out for the 1D $t-J$ model,$\underline{40}$ would be of great interest.

\section{ACKNOWLEDGMENTS}

This work was supported by DFG through SFB 652 .
1 D. M. Edwards, Physica B 378-380, 133 (2006).

2 D. M. Edwards, S. Ejima, A. Alvermann, and H. Fehske, J. Phys. Condens. Matter 22, 435601 (2010).

3 M. Berciu, Physics 2, 55 (2009).

4 L. G. L. Wegener and P. B. Littlewood, Phys. Rev. B 66, 224402 (2002).

5 K. Wohlfeld, A. M. Oleś, and P. Horsch, Phys. Rev. B 79, 224433 (2009).

6 A. Alvermann, D. M. Edwards, and H. Fehske, Phys. Rev. Lett. 98, 056602 (2007).

7 G. Wellein, H. Fehske, A. Alvermann, and D. M. Edwards, Phys. Rev. Lett. 101, 136402 (2008).

8 S. Ejima, G. Hager, and H. Fehske, Phys. Rev. Lett. 102, 106404 (2009).

9 S. Ejima and H. Fehske, Phys. Rev. B 80, 155101 (2009).

10 I. G. Bednorz and K. A. Müller, Z. Phys. B 64, 189 (1986).

11 E. Dagotto, Rev. Mod. Phys. 66, 763 (1994).

12 G. H. Jonker and J. H. van Santen, Physica 16, 337 (1950).

13 E. Dagotto, Nanoscale Phase Separation and Colossal Magnetoresistance: The Physics of Manganites and Related Compounds (Springer, Heidelberg, 2003).

14 A. Weiße and H. Fehske, New J. Phys. 6, 158 (2004).
15 R. Saito, G. Dresselhaus, and M. S. Dresselhaus, Physical Properties of Carbon Nanotubes (Imperial College Press, London, 1998).

16 H. Mousavi and M. Bagheri, Physica E 44, 1722 (2012).

17 K. S. Novoselov, A. K. Geim, S. V. Morozov, D. Jiang, Y. Zhang, S. V. Dubonos, I. V. Grigorieva, and A. A. Firsov, Science 306, 666 (2004).

18 T. Stauber and N. M. R. Peres, J. Phys. Condens. Matter 20, 055002 (2008).

19 R. J. H. Clark, in Advances in Infrared and Raman Spectroscopy, edited by R. J. H. Clark and R. E. Hester (Wiley Heyden, New York, 1984), vol. 11, p. 95.

20 D. Baeriswyl and A. R. Bishop, Phys. Scr. T19, 239 (1987).

21 S. R. White, Phys. Rev. Lett. 69, 2863 (1992).

22 E. Jeckelmann and S. R. White, Phys. Rev. B 57, 6376 (1998).

23 E. Jeckelmann, Phys. Rev. B 66, 045114 (2002).

24 E. Jeckelmann and H. Fehske, Riv. Nuovo Cimento 30, 259 (2007).

25 K. W. Becker, A. Hübsch, and T. Sommer, Phys. Rev. B 66, 235115 (2002); S. Sykora, A. Hübsch, K. W. Becker, 
G. Wellein, and H. Fehske, Phys. Rev. B 71, 045112 (2005)

26 S. Sykora, K. W. Becker, and H. Fehske, Phys. Rev. B 81, 195127 (2010).

27 J. Bonča, S. A. Trugman, and I. Batistić, Phys. Rev. B 60, 1633 (1999).

28 A. Alvermann, D. M. Edwards, and H. Fehske, J. Phys. Conf. Ser. 220, 012023 (2010).

29 C. L. Kane, P. A. Lee, and N. Read, Phys. Rev. B 39, 6880 (1989).

30 G. Martinez and P. Horsch, Phys. Rev. B 44, 317 (1991).

31 S. A. Trugman, Phys. Rev. B 37, 1597 (1988).

${ }^{32}$ V. J. Emery, S. A. Kivelson, and H. Q. Lin, Phys. Rev. Lett. 64, 475 (1990).

33 M. Dzierzawa, in The Hubbard Model, edited by D. Baeriswyl (Plenum, New York, 1995), vol. 343 of NATO
Advanced Study Institutes, Ser. B.

34 S. Ejima, F. Gebhard, and S. Nishimoto, Europhys. Lett. 70, 492 (2005).

35 T. Giamarchi, Quantum Physics in One Dimension (Clerendon Press, Oxford, 2003).

36 M. Ogata, M. U. Luchini, S. Sorella, and F. F. Assaad, Phys. Rev. Lett. 66, 2388 (1991).

37 A. Moreno, A. Muramatsu, and S. R. Manmana, Phys. Rev. B 83, 205113 (2011).

38 M. Berciu and H. Fehske, Phys. Rev. B 82, 085116 (2010).

39 I. Bloch, J. Dalibard, and W. Zwerger, Rev. Mod. Phys. 80, 885 (2008).

40 A. Eckardt and M. Lewenstein, Phys. Rev. A 82, 011606 (2010). 